ArtefaCToS. Revista de estudios de la ciencia y la tecnología

eISSN: $1989-3612$

Vol. 9, No. 1 (2020), 2. ${ }^{a}$ Época, 159-174

DOI: https://dx.doi.org/10.14201/art202091159174

\title{
Los límites de las Ciencias de la Documentación: Límites de objetivos, procesos y resultados
}

\author{
The Limits of Information Science: Limits of Aims, Processes and \\ Results
}

\author{
Antonio BEREIJO \\ Universidad de A Coruña, España \\ antonio.bereijo@udc.es
}

Recibido: 23/01/2020. Revisado: 15/02/2020. Aceptado: 25/03/2020

\section{Resumen}

El problema de las Ciencias de la Documentación puede analizarse siguiendo varios pasos. Primero, está el ángulo de análisis, que puede orientarse en dos direcciones: a) considerar estas Ciencias desde sus fronteras como quehacer científico (como una actividad científica en vez de no científica); y b) enfocar estas disciplinas desde sus posibles confines en el contexto actual y en el futuro.

Segundo, los límites de las Ciencias de la Documentación también dependen de su propio estatus científico. Así, este artículo caracteriza estas disciplinas como Ciencias Aplicadas de Diseño, es decir, como un tipo de Ciencia de lo Artificial orientada a la solución de problemas concretos en el ámbito de los archivos, bibliotecas y centros de documentación. Esta caracterización científica conlleva el análisis de los límites de los elementos estructurales y dinámicos en cuanto que Ciencias de lo Artificial, como son los objetivos, los procesos y los resultados.

Tercero, además de aspectos endógenos de esta tarea de resolución de problemas en un dominio artificial (como es comúnmente el caso de los objetivos, procesos y resultados), los límites de las Ciencias de la Documentación también dependen de los rasgos exógenos, que son aquellos elementos (sociales, culturales, etc.) que rodean este quehacer científico.

Palabras clave: fronteras; confines; diseño; endógena; exógena; artificial. 


\begin{abstract}
The problem of information science can be analyzed following several steps. First, there is the issue of the angle of analysis, which can be oriented in two directions: a) to consider information science from the viewpoint of its boundaries as scientific endeavor (i.e., as a scientific rather than a non-scientific activity); and $b$ ) to focus information science from the perspective of its possible confines in the present context and in the future.

Second, the limits of information science also depend on its own scientific status. Thus, this paper characterizes information science as an applied science of design, i.e., as a kind of science of the artificial oriented toward the solution of concrete issues in the sphere of archives, libraries, and centers of documentation. This characterization as applied science of design involves the analysis of limits of the structural and dynamic elements of this science of the artificial, such as aims, processes, and results.

Third, besides the endogenous aspects of this problem-solving task in an artificial domain (which are commonly the case of aims, processes, and results), the limits of information science also depend on exogenous features, which are those elements (social, cultural, economic, political, etc.) that surround this scientific endeavor.
\end{abstract}

Keywords: boundaries; confines; design; endogenous; exogenous; artificial.

\title{
1. Introducción
}

Se analizan aquí diversos aspectos relacionados con los límites de las Ciencias de la Documentación ${ }^{1}$. En primer lugar, se explicitan los ángulos desde los que cabe abordar el problema de los límites en estas disciplinas, que han tenido un indudable desarrollo en las últimas décadas. En segundo término, se profundiza en su vertiente de Ciencias de lo Artificial, que lleva a considerar los límites respecto de los objetivos, los procesos y los resultados, en cuanto que son Ciencias Aplicadas de Diseño. En tercera instancia, se hacen algunas reflexiones sobre los condicionantes generales que limitan las Ciencias de la Documentación. Son los elementos endógenos y los componentes exógenos.

\footnotetext{
${ }^{1}$ Hay numerosas publicaciones sobre cómo entender los estudios sobre la información, en general, y las Ciencias de la Documentación, en particular. Entre esos trabajos previos acerca de estas disciplinas son de interés los textos de Borko (1968); Cronin (2012); Griffith (1980); Holland, (2008); y Spink (2000).
} 


\section{2. Ángulos del problema de los límites científicos}

El problema de los límites de las Ciencias de la Documentación (Information Science) puede ser abordado desde distintos ángulos. Destacan dos por su carácter general: (i) los contornos que las perfilan como disciplinas científicas y (ii) los confines hasta donde pueden llegar en cuanto saberes científicos orientados a resolver problemas concretos. Porque, en primer lugar, hay que perfilar los lindes que permiten distinguir a las Ciencias de la Documentación de las meras técnicas o prácticas profesionales acumuladas (esto es, los conocimientos basados en la experiencia personal o en conocimientos compartidos por un grupo profesional, que puede ser más o menos amplio). Y, en segundo término, es preciso pensar en cuál puede ser el techo alcanzable en las tareas de procesamiento de la información y reidentificación documental en Archivos, Bibliotecas y Centros de Documentación ${ }^{2}$.

Acerca del primer aspecto — los lindes o contornos para perfilar su carácter científico- - se ha de considerar que las Ciencias de la Documentación pueden entenderse como disciplinas duales. Porque se sitúan en el campo de lo artificial - amplían las potencialidades humanas para el acceso y recuperación de la información ${ }^{3}$ al tiempo que son saberes de un entorno social, donde los usuarios demandan cada vez nuevas prestaciones, progresivamente más sofisticadas ${ }^{4}$. También cabe aceptar su presentación dentro de la categoría de Ciencia Aplicada, por cuanto es un tipo de Ciencia de Diseño — está dentro del ámbito artificial— y está orientada a objetivos, procesos y resultados, dentro de la búsqueda de soluciones a problemas concretos.

Paralelamente, sobre el segundo aspecto —el límite terminal o confines alcanzables_-, hay que advertir la existencia de complejidad en las Ciencias de la Documentación ${ }^{5}$, debido no solo al carácter dual —artificial y social— señalado, sino también a la interrelación con otras disciplinas ${ }^{6}$. El caso más obvio es la Informática (Computer Sciences), debido a la necesidad de sistemas de procesa-

\footnotetext{
2 Por "reidentificación" se entiende el poder volver a identificar algo previamente identificado. Esto supone elementos de lenguaje, conocimiento y realidad, de manera que intervienen componentes semánticos, epistemológicos y ontológicos. Sobre el concepto de "reidentificación", que tiene raíces filosóficas, puede verse González (1986).

${ }^{3}$ Ciencias de lo Artificial se entiende aquí en la acepción que acuñó Herbert A. Simon. La expresión más clara está en Simon (1996). Sobre el papel de la racionalidad en estas disciplinas científicas, véase Bereijo (2003b).

${ }^{4}$ Sobre las Ciencias de la Documentación como "Ciencias duales" - Ciencias de lo Artificial y Ciencias Sociales_, véase Bereijo (2011). Acerca de los rasgos de las Ciencias de Diseño como Ciencias de la Complejidad, cfr. Gonzalez (2013a).

${ }^{5}$ El propio concepto de "información" se ha asociado a la idea de complejidad, cfr. Bawden (2007). Acerca de la existencia de complejidad en las Ciencias de la Documentación, véase Broughton, Hansson, Hjørland, y López-Huertas (2005); Mukherjee (2008); y Vakkari (1999).

6 Sobre la caracterización de la "complejidad", cfr. Gell-Mann (1995); Gershenson and Heyliguen (2004); Heylighen (1991 y 1999); Israel (2005); y Lyons (2005).
} 
miento de información automatizados. Pero también se encuentra en el nexo con campos temáticos como la Lingüística, la Filosofía, la Historia, etc. Así, su techo científico no depende solo de la capacidad "interna” o específica de las Ciencias de la Documentación sino también de la red de dependencias que se establecen con otras disciplinas, dentro de una interdisciplinariedad epistemológica y metodológica $^{7}$ (que no una mera multidisciplinariedad) ${ }^{8}$.

Estos ángulos del problema de los límites cobran mayor relieve cuando se aprecia que es un asunto que tiene relevancia para múltiples facetas. Unas son científicas, tales como la profundidad alcanzable en investigación en este campo temático o los confines de los objetivos que busca, los procesos que despliega y los resultados que se obtienen o pueden lograr. Mientras que otras facetas son académicas, tales como la configuración de los planes de estudio, o modulan la propia actividad profesional de los agentes en los Archivos, Bibliotecas y Centros de Documentación, además de condicionar los instrumentos que permiten desarrollar las prestaciones a los usuarios (individuos, grupos u organizaciones) ${ }^{9}$.

\section{El problema de los límites de las Ciencias de la Documentación en cuanto que Ciencias Aplicadas de Diseńo}

Donde se entrecruzan los ángulos mencionados - los lindes y los confineses en el análisis de las Ciencias de la Documentación como Ciencias de lo Artificial y, en concreto, como Ciencias Aplicadas de Diseńo. Por un lado, ahí se pone de relieve la existencia de un proceso de "cientificación"10, por el que una serie de prácticas profesionales han sido reconducidas a diseños de carácter científico, dando lugar a modelos orientados hacia una articulación de objetivos, procesos y resultados. Y, por otro lado, el techo epistemológico y metodológico de las Ciencias de la Documentación viene dado precisamente por su capacidad de elaborar modelos capaces de resolver problemas cada vez más sofisticados ${ }^{11}$. Estos modelos de los diseños aplicados son demandados por usuarios con aspiraciones

\footnotetext{
${ }^{7}$ Acerca del tema de la interdisciplinariedad, cfr. Holland (2008); Moran (2006); Repko (2005); Rogers, Scaife, and Rizzo (2003); e Youngblood (2007).

${ }^{8}$ Se asume aquí que hay límites para un posible método científico universal, límites debidos a la complejidad de los fenómenos estudiados. Cfr. González (2013).

${ }^{9}$ Reflexiones sobre cómo los usuarios pueden modular la información en el entorno del ciberespacio se encuentran en Nicholas (2012).

${ }^{10}$ Acerca del concepto de "cientificación" y los rasgos de la Ciencia Aplicada, véase Niiniluoto (1993).

${ }^{11}$ En esto hay puntos de similitud con la Economía Aplicada, en cuanto que es una Ciencia de Diseño dentro del marco de las Ciencias de lo Artificial, cfr. Gonzalez (2008).
} 
que, progresivamente, se plantean como maximizadoras (al menos respecto del futuro) o bien optimizadoras para determinados fines ${ }^{12}$.

Tanto en el ángulo de los lindes como en el que atañe a los confines, hay que pensar que en estas Ciencias de Diseño hay al menos tres niveles de racionalidad en liza ${ }^{13}:$ a) el general de la Ciencia, en cuanto que hay una racionalidad científica que puede ser compartida por las diversas Ciencias; b) el específico de las Ciencias de la Documentación, en cuanto una tarea de racionalidad encaminada a la resolución de los problemas concretos que se plantean en Archivos, Bibliotecas y Centros de Documentación; y c) la racionalidad de los agentes, quienes han de tomar decisiones con criterios racionales, para lo que han de utilizar los conocimientos, prácticas y valores propios de las actividades científicas, en general, y documentales, en particular.

En la actualidad, las Ciencias de la Documentación se configuran como un conjunto de disciplinas que están orientadas a la resolución de problemas prácticos. Habitualmente, son problemas asociados a la organización del conocimiento documentado: versan comúnmente sobre aquello que se encuentra registrado por medio de un sistema de representación en un tipo de soporte ${ }^{14}$. Así, cuando se busca establecer los límites de las Ciencias de la Documentación en cuanto que Ciencia Aplicada de Diseño, el intento de establecer los límites como barreras o lindes requiere distinguir los componentes que tienen una índole científica frente a los elementos que no reúnen los rasgos de Ciencia. Esto incide después en los límites terminales o fronteras que conforman el techo de este ámbito disciplinar, que es ciertamente de naturaleza compleja ${ }^{15}$.

Cuando las Ciencias de la Documentación se caracterizan como Ciencias Aplicadas de Diseño, se puede hacer según un doble enfoque: a) desde un punto de vista histórico; y b) desde una perspectiva temática (Bereijo, 2011). Por lo que respecta al primer enfoque, las Ciencias de la Documentación están orientadas desde sus inicios a conseguir objetivos que presentan habitualmente una índole eminentemente práctica. Esta afirmación puede reforzarse por el hecho de estar asociadas las Ciencias de la Documentación a tipos específicos de Tecnologías. Así, los límites en las Ciencias de la Documentación hay que buscarlos en función de esos tres parámetros mencionados: (i) en lo que atañe a sus objetivos; (ii) en cuanto a los procesos que despliega; y (iii) en relación con los resultados que se obtienen actualmente o se pueden alcanzar en el futuro.

\footnotetext{
${ }^{12}$ Nicholas Rescher distingue entre "maximización" (obtener lo máximo posible) y "optimización” (el logro de aquello que resulta óptimo para alguien en un contexto dado). Cfr. Rescher (1987).

${ }^{13}$ Acerca de estos niveles en liza en las Ciencias de Diseño, véase González (2007).

${ }^{14}$ Es un tipo de conocimiento que se expresa en un universo documental que va más allá de los soportes físicos analógicos convencionales, Cfr. Lyman and Varian (2003).

${ }^{15}$ La naturaleza compleja de las Ciencias de la Documentación es el punto de partida para el análisis en Bereijo (2012).
} 


\subsection{Límites a tenor de los objetivos}

Sobre los objetivos hay que resaltar que giran normalmente en torno a la resolución de problemas prácticos relacionados con procesos informativos. No se busca, en rigor, un conocimiento propio de la Ciencia Básica, como es el encaminado a explicar más o a predecir mejor. Sucede, además, que la historicidad es un factor siempre presente ${ }^{16}$, que puede incidir como límite en las Ciencias de la Documentación. Porque los objetivos siempre están sometidos a una componente temporal de índole histórica. A esto se une otro factor que limita cara el futuro: las Ciencias de la Documentación utilizan con frecuencia elementos que ella misma no produce: tiene una fuerte dependencia de ámbitos disciplinares ajenos, como son la Informática, la Matemática, la Lingüística, la Sociología, etc. ${ }^{17}$.

Como ocurre habitualmente en el caso de las Tecnologías — con las que tienen estas disciplinas científicas una estrecha dependencia, sobre todo con las TIC—, los objetivos de las Ciencias de la Documentación son siempre fruto de su tiempo - presentan historicidad en su formulación y desarrollo- y surgen de la necesidad de resolver problemas concretos. Por eso, están sometidos a cambios continuos, tanto por razones internas como por la dependencia del entorno externo. De hecho, las Ciencias de la Documentación utilizan constantemente soportes tecnológicos que modulan siempre la posibilidad de conseguir objetivos propuestos. Esto significa que siempre han dispuesto de un conjunto de Tecnologías asociadas que frecuentemente tienen un carácter instrumental.

Así pues, de los límites que atañen a los objetivos de las Ciencias de la Documentación, unos son de carácter interno mientras que otros son de indole externa. Con respecto a los primeros, suelen estar vinculados a la información grabada en algún tipo de soporte documental. Comúnmente, tienen que ver con alguno de los siguientes aspectos: a) con los procesos de selección de la información documentada; b) con las vías y los mecanismos de acceso a la información grabada; c) con la gestión de registros informativos y con su posterior reidentificación; d) con las fórmulas y con los mecanismos de distribución de información a los agentes humanos que actúan como consumidores de información; y e) con el estudio de los mecanismos de la comunicación científica (Bibliometría-Cienciometría) y de los perfiles de usuarios.

Paralelamente, hay una serie de límites que tienen su origen en factores externos, donde no solo están la dependencia de los usuarios y las instituciones — cada vez más exigentes en sus demandas—, sino ante todo por la necesidad de

\footnotetext{
${ }^{16}$ Sobre el papel de la historicidad en la Ciencia, cfr. Gonzalez (2011).

${ }^{17}$ Pero esta situación no se da a la inversa, es decir, las Ciencias de la Documentación muestran una muy escasa capacidad para incorporar su propia investigación científica a otros campos de conocimiento. Tefko Saracevic afirma que los avances en las Ciencias de la Documentación (Information Science) tienen un impacto muy limitado en otros campos. Cfr. Saracevic (1997, pp. 16-27, en especial, p. 25).
} 
utilización de Tecnologías. Con ellas hay una creciente diversidad de formatos documentales. En efecto, desde la década de los años 90 del pasado siglo XX, el rápido desarrollo de las Tecnologías de la Información y la Comunicación (TIC), especialmente a través de Internet — el soporte tecnológico de la Red de redesy de la $\mathrm{Web}^{18}$, se han realizado importantes cambios. Pero son avances — con sus correspondientes límites- que tienen su origen fuera del genuino campo documental.

Como las Ciencias Documentales buscan objetivos de índole práctica, para conseguir esos fines hace falta procesos que se apoyan en teorías capaces de resolver problemas concretos. Así, según Paul Thagard, una teoría no es científica en los siguientes casos: a) cuando la teoría muestra menos progreso (progressive) que las teorías alternativas durante un largo período de tiempo, de modo que presenta problemas no resueltos; $\mathrm{y} b$ ) cuando la comunidad de profesionales (practitioners) se esfuerza poco en desarrollar la teoría para poder ofrecer soluciones a los problemas, de manera que no tiende a evaluar la teoría en relación con otras y es selectiva a la hora de considerar confirmaciones y descartes (disconfirmations) (cfr. Thagard 1978, 228). Esta doble distinción parece señalar un criterio de demarcación que atañe a las Ciencias de la Documentación, en cuanto que están concebidas como Ciencias Aplicadas de Diseño, de modo que han desarrollado teorías orientadas a la resolución de problemas prácticos.

\subsection{Límites debidos a procesos}

Ciertamente los límites debidos a procesos conectan con los límites relacionados con los objetivos. Sucede, además, que los procesos en las Ciencias de la Documentación dependen, por un lado, de otras disciplinas científicas, donde destaca la Informática (Computer Sciences); y, por otro lado, tienen una evidente dependencia las Tecnologías basadas en Internet, en cuanto que Red de índole general, y en la Web, en particular. Lo primero - las relaciones interdisciplinares- contribuyen a incrementar la complejidad del ámbito de conocimiento de las Ciencias de la Documentación. En este sentido, Wang ha señalado que las relaciones entre las Ciencias de la Documentación y la Informática se han vuelto hoy más complejas que nunca (cfr. Wang, 2009). Esto se aprecia, por ejemplo, en las tareas de gestión del conocimiento, de minería de datos o de reutilización de información. Además, acerca de las relaciones con las Tecnologías de la Información y la Comunicación (TIC), cabe afirmar que son en términos de condiciones de posibilidad para muchos procesos de las Ciencias de la Documentación (Information Science).

\footnotetext{
${ }^{18}$ Sobre la dinámica de crecimiento de la Web, cfr. Huberman y Adamic (1999). Acerca del papel de la complejidad en la Web, véase Tredinnick (2009).
} 
Aquí, al tratar de los procesos, también se dirime el carácter científico de las Ciencias de la Documentación. Cuando están desarrolladas, estas disciplinas ofrecen soluciones a problemas concretos, tras evaluar teorías y contrastar procesos de investigación, que han llevado a fórmulas más eficaces y eficientes para alcanzar los objetivos propuestos. Con todo, hay ámbitos de conocimiento dentro de las Ciencias de la Documentación que pueden considerarse científicos frente a otros que, por ahora, no son más que mera "técnica" basada en una praxis profesional acumulada ${ }^{19}$. Los ámbitos disciplinares como la Organización de Conocimiento (cfr. Broughton, Hansson, Hjørland, y López-Huertas, 2005) o la Bibliometría (cfr. Pritchard, 1969) han adquirido ya un estatuto científico. Porque han realizado una intensa tarea de elaboración de teorías orientadas a prescripciones para la resolución de problemas prácticos, que han dado lugar a instrumentos orientados a elaborar pautas específicas para conducir la actividad profesional.

En efecto, como son disciplinas "aplicadas", para tener carácter científico, han de combinar predicción y prescripción (Bereijo 2003a, 2007a y 2007b). Esto da lugar a los consiguientes procesos, para conocer el futuro posible y para dar pautas de actuación en la solución de los problemas que se plantean ${ }^{20}$. Así, los límites de las Ciencias de la Documentación derivan de su capacidad para el desarrollo de su propio estatuto epistemológico como Ciencias Aplicadas de Diseño. Sus límites están asociados a dos tipos de condiciones: I) las genuinamente internas, que están habitualmente vinculadas al avance del conocimiento para dar alcance a los objetivos propuestos (donde intervienen las predicciones y las prescripciones); y II) los factores de índole externa, que están vinculados a dar satisfacción a las demandas informativas de los usuarios. Estas nuevas demandas suelen estar vinculadas a cambios en el entorno, normalmente motivados por nuevos avances tecnológicos en lo que atañe al almacenamiento y distribución de información.

Pero también hay que saber deslindar lo que es "Ciencia Aplicada" respecto de lo que es una "aplicación de la Ciencia" (Gonzalez 2013c), que consiste en el uso del conocimiento científico en contextos bien delimitados. Así, en el caso de la Organización de Conocimiento, no es igual la tarea de diseño y desarrollo de Tesauros o de sistemas de clasificación alfanuméricos que su aplicación práctica. Esta distinción es también observable en el caso de las tareas de catalogación formal: es distinta la actividad de elaboración de estándares catalográficos que su aplicación práctica. Lo mismo ocurre con actividades del ámbito de la Bibliometría, cuando se conciben como un conjunto de métodos para analizar cuantita-

\footnotetext{
${ }^{19}$ Se trataría de una mera práctica profesional que, eventualmente, se apoya en la tradición, pero sin criterios téoricos que articulen predicción con prescripción. Sobre el papel de la predicción y la prescripción en las Ciencias de la Documentación como Ciencias Aplicadas, véase Bereijo (2003a, 2007a and 2007b).

${ }^{20}$ En esto se parece a la Economía en cuanto Ciencia Aplicada. Sobre este asunto, véase Gonzalez (1998). Acerca del caso de estas disciplinas, cfr. Bereijo (2011).
} 
tivamente la literatura científica y tecnológica o, en palabras de Alan Pritchard, como "la aplicación de métodos matemáticos y estadísticos a los libros y a otros media" (Pritchard, 1969, 348-349). Esto es distinto al quehacer de quienes usan ese conocimiento: la mera aplicación de la estadística (beam-counters) no hace que un campo de conocimiento sea científicamente riguroso.

Cabe señalar que, desde un punto de vista histórico, el nacimiento de las Ciencias de la Documentación se produjo como respuesta a la ineficiencia de la Biblioteconomía en cuanto al tratamiento documental eficaz de una tipología especial de documentos, como es la literatura científica, y realizada sobre unos perfiles de usuarios muy concretos: quienes se dedicaban a la investigación científica. De ese modo, la Biblioteconomía dejó de ser útil en el procesamiento documental de este tipo de literatura especializada.

Actualmente parece que asistimos a una repetición del fenómeno, pues cabe que los nuevos desarrollos tecnológicos se traduzcan en procesos de desintermediación (cfr. Nicholas, 2012), de modo que desplacen a los actuales perfiles profesionales que operan en este campo de conocimiento. Hay varias razones para ello: a) el impacto de Tecnologías habitualmente asociadas a la Web y a las telecomunicaciones, que evolucionan rápidamente hacia la satisfacción del usuario final (Tecnologías Front-End/User-Friendly) en términos de "accesibilidad" o "usabilidad"; b) el incremento del volumen de información en páginas web; c) la multiplicidad de tipologías documentales de índole electrónica; y d) la ubicuidad de recursos informativos.

\subsection{Límites en cuanto a los resultados}

Los límites en cuanto a los resultados o "productos" de las Ciencias de la Documentación dependen de la viabilidad de los procesos planteados y de los objetivos propuestos. Aquí, de nuevo, intervienen los límites de la Ciencia, en general, y de los agentes que hacen Ciencias de la Documentación, en particular. Su racionalidad es normalmente limitada (bounded rationality) ${ }^{21}$, en lugar de ser racionalidad maximizadora (cfr. Bereijo 2003). A este respecto, hay que resaltar que estas disciplinas despliegan una amplia variedad de procesos para alcanzar los objetivos propuestos. Esto puede verse con claridad en uno de los ámbitos más relevantes las Ciencias de la Documentación: la Organización del Conocimiento. Porque en él se despliegan procesos documentales modulados habitualmente por medio de estándares con los que se buscan importantes resultados, como son la organización, la reidentificación, el acceso y la distribución de la información documentada.

${ }^{21}$ Sobre la racionalidad limitada en el contexto de las Ciencias Sociales, véase Simon (2000 [2003]). Acerca de racionalidad científica, en general, cfr. Rescher (1999). 
Este tipo de procesos están sometidos a límites de diversa índole. Atañen a los siguientes elementos: (i) la amplia heterogeneidad y el volumen de los documentos primarios, en crecimiento exponencial (es decir, su amplia diversidad en cuanto a tipología, a su creciente volumen, etc.); (ii) a los agentes humanos que realizan procesos documentales, pues utilizan procedimientos que, en muchas ocasiones, presentan una naturaleza de elevada complejidad intelectual; (iii) a los instrumentos utilizados, que habitualmente son de base tecnológica (tales como bases de datos, sistemas de codificación, etc.), pero donde hay también elementos de índole prescriptiva, que han sido elaborados para garantizar la obtención de productos documentalmente homogéneos; y (iv) a los límites impuestos por las demandas de los consumidores finales (con frecuencia, organizaciones). Sus peticiones están habitualmente sometidas a cambios en el tiempo, de modo que varían ampliamente en función de multitud de parámetros. En muchos casos, son perfiles distintos y varían a tenor de parámetros culturales. Normalmente llevan a la adquisición de nuevas destrezas en el manejo de dispositivos informáticos.

Si el análisis lo hacemos en términos ontológicos, de modo que vemos a la Ciencia como una actividad humana entre otras, encaminada a metas cada vez más sofisticadas ${ }^{22}$, entonces los resultados se pueden ver del modo siguiente. En primer lugar, el resultado de la actividad científica en el campo las Ciencias Documentales se muestra en soluciones concretas a problemas planteados en la actualidad o que se puedan plantear en el futuro. En segundo término, como es una Ciencia Aplicada y está apoyada en diseńos, se retroalimenta de problemas que plantea la actividad profesional, con su amplia tipología de unidades de información. En tercer lugar, debido a que es un ámbito empírico, las soluciones dadas a los problemas planteados han de ser contrastadas para juzgar su validez. Así, este campo de investigación ha de poder dar soluciones potencialmente universales o universalizables ${ }^{23}$, en lugar de proporcionar meras soluciones ad hoc o una pura resolución de casos concretos sin proyección ulterior.

\section{Sobre los condicionantes generales que limitan las Ciencias de la Docu- mentación: Elementos endógenos y componentes exógenos}

Hay dos grandes tipos de condicionales generales limitantes para las Ciencias de la Documentación: los elementos endógenos, que son los resaltados hasta ahora (la relación entre objetivos, procesos y resultados), y los componentes exógenos, que son aquellos que rodean la actividad de estas disciplinas, que están en un entorno cultural y social históricamente cambiante. En cuanto a los elementos endóge-

\footnotetext{
${ }^{22}$ Acerca de los caracteres de la Ciencia, en general, y en torno a la Ciencia como actividad humana, véase Gonzalez (2005, 3-49; en especial, 9-11); y Gonzalez (2013).

${ }^{23}$ Con todo, conviene advertir que hay límites al universalismo metodológico, cfr. Gonzalez (2012).
} 
nos, destacan las limitaciones en la capacidad de computación de la información, otros límites debidos a ser una tarea realizada por agentes humanos y los límites tecnológicos que les condicionan, que derivan de los instrumentos tecnológicos utilizados por los documentalistas para el procesamiento y transmisión de la información.

Respecto de los componentes exógenos - los límites desde un punto de vista externo-, destacan los papeles jugados por los usuarios y los cometidos desempeñados por las instituciones, además de las regulaciones existentes en cada momento (de índole local, regional, estatal o internacional). De este modo, la clave no es la práctica profesional en cuanto tal, sino los límites mismos de una actividad apoyada en un conocimiento científico aplicado, que es proyectado hacia el futuro mediante la predicción y que está modulado por las pautas de prescripción para solucionar problemas.

Desde un punto de vista histórico, tanto las Ciencias de la Documentación como su precursora - la Biblioteconomía - surgieron para dar respuesta al problema de la organización y el acceso a la información documentada. Así, desde sus inicios, estas Ciencias Aplicadas han buscado ofrecer respuestas ante el problema de la organización y acceso a las fuentes documentales disponibles. Tenían que atender a su constante crecimiento frente a dos limites genuinamente humanos, que tienen una naturaleza interna: por un lado, las restricciones en capacidad para recordar grandes volúmenes de información (la memoria); y, en segundo término, las restricciones en cuanto a la capacidad de computación de la información disponible ${ }^{24}$.

Pero late aquí un problema de fondo. Se trata de un problema de complejidad en el proceso de interacción que se plantea entre el agente humano y el corpus documental que él mismo produce. En efecto, ese problema fundamental, que reside en los límites de las capacidades humanas, fue el origen de un campo de conocimiento específico y también lo fue de unos perfiles profesionales con rasgos propios. Esa interacción entre el agente y el documento da lugar a problemas que han perdurado en el tiempo y se ha incrementado notoriamente en los últimos 30 años, gracias a la expansión de Internet como Red de redes (en particular, las décadas de la Web abierta al público) y de sus Tecnologías asociadas. También ha incidido la multiplicidad de formatos electrónicos y de nuevas tipologías documentales que han surgido en estas décadas.

Para resolver esa complejidad, además de la elaboración de elementos conceptuales — con sus correspondientes desarrollos teóricos_-, se han puesto a punto

\footnotetext{
${ }^{24}$ Cabe recordar, a este respecto, la metáfora de la Selva Selvaggia, a la que se refería José Ortega y Gasset para aludir a la importancia de las tareas de organización documental, o la propuesta de diseño de Memex (Memory Extender), de Vannevar Bush, como precursor tecnológico de los actuales sistemas de búsqueda y reidentificación de información a partir de la interconexión de conceptos.
} 
diversas Tecnologías, para intentar resolver el problema de organización y acceso a fondos documentales. Ahora se plantea cómo poner orden, seleccionar y garantizar el acceso público de la documentación en formato electrónico, es decir, de las nuevas formas de representación del conocimiento. Han sido formuladas mediante sistemas de significación diversos y que, además de presentar un crecimiento exponencial, tiene características distintivas.

A este respecto, como en otros ámbitos científicos, se han diseñado Tecnologías que han buscado emular el funcionamiento del cerebro humano en sus procesos de almacenamiento, organización y recuperación de la información. Es aquí donde se muestra con claridad uno de sus límites externos en el ámbito de las Ciencias Documentales: la incorporación de conocimiento procedente de ámbitos diversos (Informática, Ciencias Cognitivas, Matemática) para poder dar respuesta a los problemas que busca resolver.

Esto explica que, habitualmente, muchos de los instrumentos tecnológicos que se utilizan en las Ciencias de la Documentación hayan sido elaborados para resolver problemas en otros ámbitos de conocimiento. Es el caso, por ejemplo, de las primeras aplicaciones informáticas en el terreno bibliotecario, que no se aplicaron a las tareas documentales propiamente dichas (como la construcción de catálogos bibliográficos) sino a la automatización de los servicios de préstamo. Eran un derivado de programas informáticos comerciales que estaban orientados a la gestión de mercancías.

En el mismo sentido, las primeras tentativas de automatización de procesos de catalogación descriptiva se realizaron a partir de sistemas de diagnóstico de enfermedades sanguíneas (MICYN). Esto explica la propia índole entitativa de las Ciencias de la Documentación, que se configura como un conjunto de disciplinas y de conocimientos diversos, que se nutren de avances de campos del saber diferentes. De ahí que sus límites dependen no solo de elementos endógenos, sino también de condicionantes exógenos. A este respecto, las Ciencias de Documentación comportan interdisciplinariedad. Esto es, sin duda, fuente de complejidad, tanto estructural como dinámica ${ }^{25}$, lo que constituye un factor que modula su futuro.

\footnotetext{
${ }^{25}$ La complejidad es dual: estructural y dinámica. La primera atańe a la configuración misma epistemológica y ontológica- del sistema complejo, mientras que la segunda incumbe al cambio a través del tiempo, que en cuestiones de Ciencias de Diseño está surcado por la historicidad. Cfr. Gonzalez (2013a).
} 


\section{Referencias bibliográficas}

Bawden, David (2007). Information as Self-organized Complexity: A Unifying Viewpoint. Information Research, 12(4). http://informationr.net/ir/12-4/colis/colis31.html

Bereijo, Antonio (2003a). Predicción y prescripción en las Ciencias de lo Artificial: Incidencia en las Ciencias de la Documentación. En Wenceslao J. González (ed.), Racionalidad, historicidad y predicción en H. A. Simon (pp. 279-309). Netbiblo: A Coruña.

Bereijo, Antonio (2003b). Racionalidad en las Ciencias de lo Artificial: El enfoque de la racionalidad limitada. En Wenceslao J. González (ed.), Racionalidad, historicidad y predicción en H. A. Simon (pp. 131-146). Netbiblo: A Coruña.

Bereijo, Antonio (2007a). Caracteres de la predicción en las Ciencias de la Documentación: De la Information Science al Análisis Documental. En Wenceslao J. González (ed.), Las Ciencias de Diseño: Racionalidad limitada, predicción y prescripción (pp. 217-235). Netbiblo: A Coruña.

Bereijo, Antonio (2007b). La articulación de la prescripción en las Ciencias de la Documentación. En Wenceslao J. González (ed.), Las Ciencias de Diseño: Racionalidad limitada, predicción y prescripción (pp. 267-285). Netbiblo: A Coruña.

Bereijo, Antonio (2011). The 'Category of Applied Science:' An Analysis of its Justification from 'Information Science' as Design Science. En Wenceslao J. Gonzalez (ed.), Scientific Realism and Democratic Society: The Philosophy of Philip Kitcher (pp. 327-350). Poznan Studies in the Philosophy of Science and the Humanities. Amsterdam: Rodopi.

Bereijo, Antonio (2012). La sobriedad de factores en las Ciencias de la Documentación (Information Science) en cuanto Ciencias de la Complejidad: Proyección en el Análisis Documental. En Wenceslao J. González (ed.), Las Ciencias de la Complejidad: Vertiente dinámica de las Ciencias de Diseño y sobriedad de factores (pp. 267-287). Netbiblo: A Coruña.

Borko, Harold (1968). Information Science: What is it? American Documentation, 19(1), 3-5.

Broughton, Vanda, Hansson, Joacim, Hjørland, Birger and López-Huertas, María J. (2005). Knowledge Organization. En Leif Kajberg and Leif Lørring (eds.), European Curriculum Reflections on Library and Information Science Education (pp. 133-149). Copenhage: Royal School of Library and information Science.

Cronin, Blaise (2012). The Waxing and Waning of a Field: Reflections on Information Studies Education. En Information Research, 17(3), 1-9. http://information r.net/ ir/17-3/paper529.html

Gell-Mann, Murray (1995). What's Complexity? Complexity, 1(1), 16-19.

Gershenson, Carlos y Heyliguen, Francis (2004). How Can We Think the Complex? En Kurt A. Richardson (ed.), Managing the Complex: Philosophy, Theory, and Practice 
(pp. 1-13). Naples FL: Institute for the Study of Coherence and Emergence/ Information Age Publishing.

González, Wenceslao J. (1986). La Teoría de la Referencia. Strawson y la Filosofía Analítica. Salamanca-Murcia: Ediciones Universidad de Salamanca y Publicaciones de la Universidad de Murcia.

Gonzalez, Wenceslao J. (1998). Prediction and Prescription in Economics: A Philosophical and Methodological Approach. Theoria: An International Journal for Theory, History and Foundations of Science, 13(32), 321-345.

Gonzalez, Wenceslao J. (2005). The Philosophical Approach to Science, Technology and Society. En Wenceslao J. Gonzalez (ed.), Science, Technology and Society: A Philosophical Perspective (pp. 3-49). A Coruña: Netbiblo.

González, Wenceslao J. (2007). Configuración de las Ciencias de Diseño como Ciencias de lo Artificial: Papel de la Inteligencia Artificial y de la racionalidad limitada. En Wenceslao J. González (ed.), Las Ciencias de Diseño: Racionalidad limitada, predicción y prescripción (pp. 41-69). A Coruña: Netbiblo.

Gonzalez, Wenceslao J. (2008). Rationality and Prediction in the Sciences of the Artificial: Economics as a Design Science. En Maria Carla Galavotti, Roberto Scazzieri y Patrick Suppes (eds.), Reasoning, Rationality, and Probability (pp. 165-186). Stanford: CSLI Publications.

Gonzalez, Wenceslao J. (2011). Conceptual Changes and Scientific Diversity: The Role of Historicity. En Wenceslao J. Gonzalez (ed.), Conceptual Revolutions: From Cognitive Science to Medicine (pp. 39-62). A Coruña: Netbiblo.

Gonzalez, Wenceslao J. (2012). Methodological Universalism in Science and its Limits: Imperialism versus Complexity. En Krzystof Brzechczyn y Katarzyna Paprzycka (eds.), Thinking about Provincialism in Thinking, Poznan Studies in the Philosophy of the Sciences and the Humanities, vol. 100 (pp. 155-175). Amsterdam/N. York: Rodopi.

Gonzalez, W. J. (2013a). The Sciences of Design as Sciences of Complexity: The Dynamic Trait. En Hanne Andersen, Dennis Dieks, Wenceslao J. Gonzalez, Thomas Uebel y Gregory Wheeler (eds.), New Challenges to Philosophy of Science (pp. 293305). Dordrecht: Springer.

González, Wenceslao J. (2013b). Límites del universalismo metodológico: El problema de la complejidad. Naturaleza y libertad. Revista de estudios interdisciplinares, 2, 61-89. http://www.uma.es/naturalezaylibertad/resources/Vol_2_2013/2-2013-3.pdf

Gonzalez, Wenceslao J. (2013c). The Roles of Scientific Creativity and Technological Innovation in the Context of Complexity of Science. En W. J. Gonzalez (ed.), Creativity, Innovation, and Complexity in Science (pp. 11-40). A Coruña: Netbiblo.

Griffith, Belver C. (ed). (1980). Key Papers in Information Science. N. York: Knowledge Industry Publications. 
Heylighen, Francis (1991). Coping with Complexity: Concepts and Principles for a Support System. Systemica, 8, Part 1, 39-55.

Heylighen, Francis (1999). The Science of Self-Organization and Adaptativity. En L. Douglas Kiel (ed.), The Encyclopedia of Life Support Systems (pp. 1-26). Oxford: Eolss Publishers.

Holland, George A. (2008). Information Science: An Interdisciplinary Effort? Journal of Documentation, 64(1), 7-23.

Huberman, Bernardo A. y Adamic, Lada A. (1999). Growth Dynamics of the World-Wide Web. Nature, 401(9), 131.

Israel, Giorgio (2005). The Science of Complexity: Epistemological Problems and Perspectives. Science in Context, 18(3), 479-509.

Lyman, Peter y Varian, Hall R. (2003). How much Information? http://groups.ischool. berkeley.edularchivelhow-much-info-2003/printable_report.pdf

Lyons, Michael (2005). Knowledge and the Modelling of Complex Systems. Futures, 37(7), 711-719.

Moran, Joe (2006). Interdisciplinarity. London: Routledge.

Mukherjee, Indranil (2008). Understanding Information System Failures from the Complexity Perspective. Journal of Social Sciences, 4(4), 308-319.

Nicholas, David (2012). Disintermediated, decoupled and down. En CILIP Update, 2931. http://ciber-research.euldownload/20120501-CILIP_Update_March_2012_ pp29-31.pdf

Niiniluoto, Ilkka (1993). The Aim and the Structure of Applied Research. Erkenntnis, $38(1), 1-21$.

Pritchard, Alan (1969). Statistical Bibliography or Bibliometrics. Journal of Documentation, 25(4), 349-349. http://independent.academia.edu/AlanPritchard/Papers/602982/Statistical_bibliography_or_bibliometric

Repko, Allen (2005). Interdisciplinary Practice: A Student Guide to Research and Writing. Boston: Pearson Custom.

Rescher, Nicholas (1987). Ethical Idealism: An Inquiry into the Nature and Function of Ideals. Berkeley: University of California Press.

Rescher, Nicholas (1999). Razón y valores en la Era cientifico-tecnológica. Barcelona: Paidós.

Rogers, Yvonne, Scaife, Mike y Rizzo, Antonio (2003). Interdisciplinarity: An Emergent or Engineered Proccess? En CSRP Technical Report, vol. 556. Brighton: University of Sussex.

Saracevic, Tefco (1997). Users Lost: Reflections on the Past, Future, and Limits of Information Science. Newsletter ACM SIGIR Forum, 31(2), 16-27. https://doi. org/10.1145/270886.270889 
Simon, Herbert A. (1996). The Sciences of the Artificial. Cambridge, MA: The MIT Press.

Simon, Herbert A. (2000). Bounded Rationality in Social Science: Today and Tomorrow. Mind and Society, 1(1), 25-39.

Spink, Amanda (2000). Toward a Theorethical Framework for Information Science. Special Issue on Information Science Research, 3(2), 56-84.

Thagard, Paul R. (1978). Why Astrology is a Pseudoscience. En Peter D. Asquit y Ian Hacking (eds.), Philosophy of Science Association, vol. 1, PSA, East Lansing (pp. 223-234). http//www.cavehill.uwi.esu/bnccde/PH29A/thagard.html

Tredinnick, Luke (2009). Complexity Theory and the Web. Journal of Documentation, 65(5), 797-816.

Vakkari, Pertti (1999). Task Complexity, Problem Structure and Information Actions: integrating Studies on Information Seeking and Retrieval. Information Processing and Management, 35(6), 819-837.

Wang, Bing (2009). Information Science: The Territory and Relations to Computing Disciplines. Journal of Library and Information Science, 35(1), 4-22.

Youngblood, Dawn (2007). Interdisciplinary studies and the Bridging Disciplines: a Matter of Process. Journal of Research Practice, 3(2), 1-9. http://jrp.icaap.org/ondex. php/jrplarticle/view/104/101 